\title{
A Search for Fragmentation Debris Near Ursa Major Stream Stars
}

\author{
F. C. WITTEBORN, J. D. BREGMAN, ${ }^{1}$ D. F. LESTER, ${ }^{2}$ AND D. M. RAN K ${ }^{3}$ \\ Ames Research Center, NASA, Moffett Field, California 94035
}

Received December 7, 1981; revised March 19, 1982

\begin{abstract}
Accretion models of planet formation and the early cratering history of the solar system suggest that planet formation is accompanied by a cloud of debris resulting from accumulation and fragmentation. A rough estimate of the infrared luminosities of debris clouds is presented for comparison with measured $10-\mu \mathrm{m}$ luminosities of young stars. New measurements of $13 \mathrm{~F}, \mathrm{G}$, and $\mathrm{K}$ mainsequence stars of the Ursa Major Stream, which is thought to be about $2.7 \times 10^{8}$ years old, place constraints on the amount of debris which could be present near these stars.
\end{abstract}

\section{INTRODUCTION}

Very little observational evidence has been set forth to suggest that planets are forming in the vicinity of young stars. Yet some theories of accretion of planets from cool gas and dust require on the order of $10^{8}$ years for formation of the terrestrial planets (Wetherill, 1978; Safronov, 1977) and longer for the outer planets (Safronov, 1977). The cratering history of the Moon indicates that significant accretion in the solar system was still taking place at least $5 \times 10^{8}$ years after its origin (Hartmann, 1972). Stars in this age range may be selected from open clusters whose members formed at approximately the same time and whose ages can be decided from evolutionary and kinematic studies. For example, the Ursa Major Stream stars are about $2.7 \times 10^{8}$ years old (Giannuzzi, 1979) and the Pleiades are $1.5 \times 10^{8}$ years old (Yuan and Waxman, 1977). The accretion process is inevitably accompanied by fragmentation. Accretion and fragmentation produce a debris cloud which radiates predominantly in the infrared. This infrared radiation would appear as an excess on the long-wavelength tail of the spectrum of the central star (Hartmann,

${ }^{1}$ Ames Associate.

2 National Research Council Associate.

${ }^{3}$ Lick Observatory.
1978). We present here a crude estimate of the wavelength dependence and intensity of such infrared excesses and observational results from a small sample of $F, G$, and $K$ main-sequence stars from the Ursa Major Stream.

\section{ESTIMATE OF INFRARED EMISSION FROM A DEBRIS CLOUD}

In estimating the luminosity of the debris cloud surrounding a star, we will use some assumptions implicit in theories in which planets are formed by sweeping up smaller solid particles in orbit around an already formed star. Safronov (1969) used this approach to explain the formation of the planets. The time required to form the outermost planets by this method appears to be too long, but its application to the terrestrial planets is an active field and has been reviewed recently by Wetherill (1980). The existence of a debris cloud in the vicinity of newly formed planets is not necessarily model dependent because collisions are likely in any system with a large number of orbiting objects. The cratering rate of the terrestrial planets was at least $10^{3}$ times greater $4.1 \times 10^{9}$ years ago than it is now (Hartmann, 1972). This implies a correspondingly higher number density of interplanetary particles. The calculations made here permit an estimate of the total mass of 
such particle distributions based on observed infrared luminosities.

We will examine the debris cloud resulting from the fragmentation of planetesimals in the late stages of planet formation. Since we have data for only one example, we will assume that the debris is spatially distributed like the rocky mass of the solar system. This debris is not expected to lie precisely in the ecliptic plane because of fragmentation in high-speed collisions. Thus inclinations to the ecliptic averaging $9.5^{\circ}$ or higher like the asteroids (Allen, 1973) are a reasonable expectation. For convenience we chose a model of debris that is a composite of eight toroids having major radii equal to the average distance from the Sun of each of the planets from Mercury to Neptune. The mass of debris in each toroid will be chosen in proportion to the rocky masses of each of these planets. Since these estimates are to be applied to stars on the order of $10^{8}$ years old, we will assume that the planets as far out as Jupiter have accumulated over $90 \%$ of their final mass (Safronov, 1969: Wetherill, 1978) and that the gaseous component of the nebula has already dispersed or accreted. The total mass of the debris is a free parameter which must be less than $10^{-4} M_{\odot}$ if the cloud is to be optically thin. The debris cloud mass for a given star can be estimated by comparison of its observed infrared luminosity with the luminosities calculated from our model for various values of the total mass.

In order to write an expression for the total luminosity of the debris cloud, we first note that the intensity of emitted radiation at wavelength $\lambda$ from a single spherical dust particle of radius $r$, whose distance from the star heating it is $R$, and whose distance from the observer is $S$, is given by

$$
i_{\lambda}=\epsilon(\lambda, r) B[\lambda, T(r, R)] r^{2} / S^{2},
$$

where $\epsilon(\lambda, r)$ is the emissivity, $B[\lambda, T(r, R)]$ is the Planck function, and $T$ is the temperature of the particle. The emissivity will be assumed to be 0.5 for $r>\lambda / 2 \pi$ and $\pi r / \lambda$ for $r \leq \lambda / 2 \pi$. For larger sizes, the value 0.5 is a slight underestimate based on emissivities of asteroids ranging from 0.98 to 0.6 (Morrison, 1977). Calculations of the wavelength dependence of extinction coefficients by Leung (1975) for several possible types of dust particles show long-wavelength behavior ranging from $\lambda^{-1}$ to $\lambda^{-2}$. We examine only the $\lambda^{-1}$ case since it yields cooler particles and thus yields a lower value for $10-\mu \mathrm{m}$ flux for a given set of other conditions. The temperature is determined by assuming radiative equilibrium between radiation absorbed from the star on one side of the particle and radiation emitted by the particle in all directions. Absorption efficiency and emissivity are equal at a given wavelength. Small particles will generally be hotter than large particles. This happens because the small ones are not good radiators, since the peaks of their emission spectra lie in the wavelength range $\lambda>2 \pi r$, where their emissivities are dropping as $\lambda^{-1}$. The spectrum of absorbed energy from the central star peaks at shorter wavelengths where the particle emissivities (and thus absorptivities) are higher.

In order to integrate Eq. (1) over the range of particle sizes we must know the particle size distribution. Dohnanyi (1969) derived a theoretical expression for the steady-state distribution of masses of particles in collision and showed that it matched the observed distribution of the asteroids except for the three largest ones. He found that the number of particles in the mass range $m$ to $m+d m$ is $d N(m) \sim m^{-\alpha} d m$ and that $\alpha=1.837$. If we assume that the density is independent of mass, the corresponding distribution in terms of radii of spherical particles is $d N(r)=$ constant $\times r^{-\beta} d r$, where $\beta=3.511$. The smallest particles will contribute the most to the luminosity so it is somewhat risky to extend this distribution law to submicron particle sizes without empirical evidence, particularly since additional removal mechanisms become increasingly important for the smaller particles (see Discussion). A synthesis of information on the size distribution of inter- 
planetary dust grains by Le Sergeant and Lamy (1980), based on analyses of lunar microcraters, suggests a two-component model for grains between $10^{-2}$ and $10^{3} \mu \mathrm{m}$ in diameter. They obtain a fit to radii less than $1 \mu \mathrm{m}$ with $\beta=3.85$ but with a shallower slope from 1 to $100 \mu \mathrm{m}$, gradually steepening again at larger radii. A rough fit to their curve can be made with $\beta=3.5$ from radii of 0.03 to $10^{3} \mu \mathrm{m}$. For purposes of our estimate the value 3.5 for $\beta$ will be used with the understanding that this point should be investigated more thoroughly for particular models of accretion and fragmentation. It should be emphasized that we are not assuming a relation between asteroidal debris and interplanetary dust at Earth's orbit. We view both as collisional debris and use the fact that the $\beta$ values for their size distributions are reasonably close to 3.5 . We may now write for the intensity of radiation from a ring of particles at distance $R_{3}$ from the Sun

$$
\begin{aligned}
I(\lambda, j)=\int_{r_{\min }}^{r_{\max }} \epsilon(\lambda, r) B\left[\lambda, T\left(r, R_{j}\right)\right] \\
\times \frac{r^{2}}{S^{2}} \frac{d N\left(r, R_{j}\right)}{d r} d r,
\end{aligned}
$$

where

$$
\frac{d N\left(r, R_{j}\right)}{d r}=C_{j} r^{-3.5}
$$

$C_{j}$ is determined from the mass of rocky material in each ring.

We choose one $j$ value corresponding to each planet starting with 1 for Mercury. In Table I we list the $R_{j}$ 's for each planet to be considered and the approximate rocky core masses $M_{j}$. Let $f_{j}$ be the fraction of $M_{j}$ that has not yet accreted in toroid $j$. Then the mass of the toroid is

$$
f_{j} M_{j}=\int_{r_{\min }}^{r_{\max }} \frac{4}{3} \pi r^{3} \rho \frac{d N\left(r, R_{j}\right)}{d r} d r,
$$

where $\rho$ is the density of the dust material. From (3) and (4) we have

$$
C_{j}=3 f_{j} M_{j} /\left(8 \pi \rho r_{\max }^{1 / 2}\right),
$$

when $r_{\min }$ is much less than $r_{\max }$. The largest
TABLE I

Parameters Used for Debris-Filled Toroids

\begin{tabular}{ccc}
\hline Major radius (AU) & $\begin{array}{c}\text { Rocky mass }^{a} \\
\left(10^{-6} M_{\odot}\right)\end{array}$ \\
\hline 0.387 & 0.166 \\
0.723 & 2.45 & \\
1.000 & 3.04 & \\
1.524 & 0.32 & \\
5.20 & 60 & \\
9.54 & 60 & \\
19.18 & 40 & (includes ices) \\
30.06 & 49 & (includes ices)
\end{tabular}

a The rocky core masses for the outer planets were chosen on the basis of calculations by Grossman et al. (1980) for Saturn and Jupiter and by Hubbard and McFarlane (1980) for Uranus and Neptune. Average distances from the Sun and masses of terrestrial planets are from Allen (1973).

particle radius, $r_{\max }$, is chosen to be $50 \mathrm{~km}$ to correspond roughly to the radii of the particles which produced the largest impact craters on the Moon (Wetherill, 1975). Larger planetesimals are suggested by some accretional theories (Greenberg, 1979), but it is not clear that they should be included in the distribution of debris fragments. For the density we choose $4 \mathrm{~g} / \mathrm{cm}^{3}$, which is at the upper end of the range $2-4 \mathrm{~g}$ / $\mathrm{cm}^{3}$ given by Brownlee et al. (1973). The smallest particle radius, $r_{\min }$, is assumed to be in the range 0.01 to $0.1 \mu \mathrm{m}$ on the basis of lunar microcrater size distributions (Le Sergeant and Lamy, 1980).

The total intensity is just the summation over $j$ of $I(\lambda, j)$. A convenient way to express the result is the ratio, $\Gamma(\lambda)$, of the debris cloud intensity to that of the central star. Using Eqs. (2)-(5) and the Planck function for a star of temperature $T^{*}$ and radius $r^{*}$, we have

$$
\begin{aligned}
\Gamma(\lambda) & =\sum_{j=1}^{\infty} 3 f_{j} M_{j}\left[B\left(\lambda, T^{*}\right) r_{*}^{2} 8 \pi \rho r_{\max }{ }^{1 / 2}\right]^{-1} \\
& \times \int_{r_{\min }}^{r_{\max }} \epsilon(\lambda, r) B\left[\lambda, T\left(r, R_{j}\right)\right] r^{-1.5} d r .
\end{aligned}
$$

This expression is valid only for an opti- 
cally thin cloud in which the particles are not shaded by each other. The cross-sectional radii $W_{j}$ of the toroids have not yet been discussed. For our purposes it is sufficient to use the average temperature, $T\left(r, R_{j}\right)$, for all particles of radius $r$ inside the toroid $j$. The larger $W_{j}$ is, the more material that can be placed in the toroid without making it optically thick. An idea of a reasonable value of $W_{j} / R_{j}$ can be obtained from the average inclination of asteroid orbits, $9.5^{\circ}$, or the spatial distribution of the zodiacal cloud which extends tens of degrees from the ecliptic.

Since debris near Saturn and the planets farther out contributes little to the wavelengths observationally available to us, and since Jupiter and the terrestrial planets formed in comparable times in accretion theories (Wetherill, 1978, Safronov, 1969), $f_{j}$ was assumed to be the same for all $j$ values in the evaluations of $\Gamma(\lambda)$ considered here.

Table II is a list of several values of $\Gamma(10.5 \mu \mathrm{m})$ evaluated numerically. As one

TABLE II

Calculated flux from Debris Cloud Relative to Central. Star at $10.5 \mu \mathrm{m}$ for Various

Choices of Minimum Dust Particle Radius, Debris Cloud Mass, and Star Type

\begin{tabular}{llcccc}
\hline $\begin{array}{c}r_{\text {mn }} \\
(\mu \mathrm{m})\end{array}$ & $f$ & $\begin{array}{c}f \sum M_{j} \\
\left(10^{-6} M_{\odot}\right)\end{array}$ & $\begin{array}{c}\text { Central } \\
\text { star } \\
\text { type }\end{array}$ & $\Gamma(10.5 \mu \mathrm{m})$ & $\Delta M_{\mathrm{N}}$ \\
\hline 0.01 & 0.1 & 22 & G2V & 3.7 & 1.7 \\
0.01 & 0.01 & 2.2 & G2V & 0.37 & 0.34 \\
0.1 & 0.01 & 2.2 & G2V & 0.25 & 0.24 \\
1.0 & 0.01 & 2.2 & G2V & 0.10 & 0.10 \\
0.01 & 0.01 & 2.2 & F0V & 0.49 & 0.43 \\
0.01 & 0.01 & 2.2 & F2V & 0.44 & 0.40 \\
0.01 & 0.01 & 2.2 & F5V & 0.40 & 0.37 \\
0.01 & 0.01 & 2.2 & F6V & 0.39 & 0.36 \\
0.01 & 0.01 & 2.2 & F8V & 0.39 & 0.36 \\
0.01 & 0.01 & 2.2 & G0V & 0.38 & 0.35 \\
0.01 & 0.01 & 2.2 & G8V & 0.35 & 0.33 \\
0.01 & 0.01 & 2.2 & K2V & 0.30 & 0.28 \\
\hline
\end{tabular}

Note. Column 1, $r_{\mathrm{min}}$, is the minimum particle radius in $\mu \mathrm{m}$. Column 2, $f$, is the fraction of the final planetary rocky mass that has not yet accreted. Column $3, f \Sigma M_{3}$, is the total mass of the debris cloud. Column 4 is $\Gamma(10.5 \mu \mathrm{m})$ which is the debris cloud 10.5- $\mu \mathrm{m}$ flux divided by the $10.5-\mu \mathrm{m}$ flux of the central star. Column $5, \Delta M_{\mathrm{N}}$, is the decrease in magnitude (i.e., brightening) caused by the presence of the debris cloud.

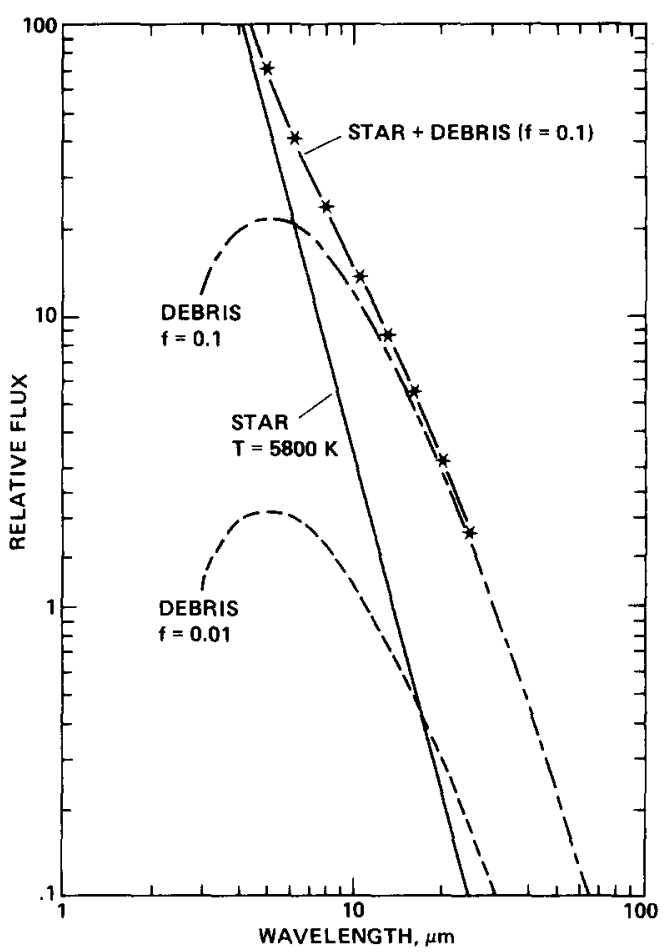

Fig. 1. Calculated spectra of debris clouds.

would expect, smaller values of $r_{\min }$ make the debris clouds easier to detect. We note that if $r_{\min }$ is $0.01 \mu \mathrm{m}$ and $f_{j}$ 's $=f=0.01$ that a significant enhancement, 0.34 magnitude, in the $10.5-\mu \mathrm{m}$ brightness of a G2V star is predicted. Even if $r_{\min }$ is $0.1 \mu \mathrm{m}$ the enhancement is a detectable 0.24 magnitude when $f=0.01$. In calculating $\Gamma(10.5 \mu \mathrm{m})$ for various stellar types we used effective star temperatures given by Johnson (1966) and luminosities and radii interpolated from values in Allen (1973). If all other parameters are kept constant, the ratio $\Gamma(10.5 \mu \mathrm{m})$ varies from about 1.3 times that of the Sun for F0V stars to 0.8 times that of the Sun for $\mathrm{K} 2 \mathrm{~V}$ stars. These estimates do not take into account differences in spatial distribution of the debris which could affect the luminosity ratios considerably.

In Fig. 1 spectra determined from the product of $\Gamma(\lambda)$ and the Planck function for a solar-type star are plotted. The relative contribution of the cloud increases at longer wavelengths, but the total intensity drops 
rapidly at wavelengths longer than about 10 $\mu \mathrm{m}$.

\section{OBSERVATIONS}

The observational approach was to measure the $\mathrm{N}$-magnitudes (corresponding to the 8.5- to 13.5- $\mu \mathrm{m}$ band) of all $\mathrm{F}, \mathrm{G}$, and $\mathrm{K}$ main-sequence stars of the Ursa Major Stream for which sensitivity was adequate to detect a debris cloud of $2 \times 10^{-5} M_{\odot}$ or smaller. The stars were selected from Roman's (1949) list of probable members. The $\mathrm{N}$-magnitudes were then compared with their known visual magnitudes and with Johnson's (1966) V-N values for normal F, $G$, and $\mathrm{K}$ main-sequence stars. Detections of infrared excesses ( $\mathrm{V}-\mathrm{N}$ greater than Johnson's values) were to be followed up by circular variable filter (CVF) spectrophotometry to determine the spectral shape of the excess in the range 8.5 to $13.5 \mu \mathrm{m}$.

Most of the photometric measurements were made with a liquid helium-cooled $\mathrm{Si}$ : As photoconductor using either of two cooled broad-band filters in the 8.5 to 13.5 $\mu \mathrm{m}$ atmospheric window. The observations of $\gamma$ Lep A and B were made in a $1-\mu \mathrm{m}$ band centered at $10.5 \mu \mathrm{m}$ using $\mathrm{Si}$ : Bi photoconductors. The measurements were calibrated against the flux measured from standard stars $\alpha$ Tau or $\alpha$ Boo through a similar air mass.

For $\alpha$ Tau the $\mathrm{N}$-magnitude was assumed to be -2.98 and for $\alpha$ Boo, -3.26 . These values are averages of magnitudes at $11 \mu \mathrm{m}$ obtained by Gillett et al. (1971) and Gehrz and Woolf (1971). The spectrum of $\alpha$ Boo was shown by Hackwell (1972) to follow a blackbody quite well throughout the range 8.5 to $13 \mu \mathrm{m}$, so use of its $11-\mu \mathrm{m}$ magnitude as a calibration in any of the three bandwidths used in our measurements is a reasonable assumption. The same spectral behavior can be expected for $\alpha$ Tau which is also a $\mathrm{K}$ giant. Most of the observations were made with the NASA/Univ. of Arizona 60-in. telescope at Mt. Lemmon. Several additional measurements were made with the 120-in. Shane telescope of the Lick
Observatory. No significant infrared excesses were found for any of the Ursa Major Stream stars observed. Those for which we could set $\mathrm{N}$-magnitudes or upper limits on $\mathrm{V}-\mathrm{N}$ within 1.7 of the normal are listed in Table III. The spectral types and visual magnitudes were taken from the Yale Catalog of Bright Stars (Hoffleit, 1964). At least five stars on the list are members of double star systems. $\eta$ Cor Bor has two components of equal brightness and spectral type. It is the only star system of the 13 observed which appears to have a detectable excess, but even this is less than two standard deviations from normal. The results may be summarized by noting that $1 \sigma$ upper limits of 0.3 magnitude or less can be put on $\mathrm{N}$ (normal) $-\mathrm{N}$ (measured) for 6 of the stars and of 1.7 magnitudes or less for all 13. The quality of the measurements varies considerably from star to star even though several attempts were made on each one. We consistently measured higher noise for objects with large negative declinations such as $\alpha$ Crv and $\gamma$ Lep. Observing near the northern limit ( $+60^{\circ}$ declination) of the Mt. Lemmon telescope also yielded higher noise, evidently because of air currents rising from the warm room which is located on the north side of the observatory. Measurements performed under improved observing conditions, or with larger telescopes, will undoubtedly reduce these limits further.

\section{DISCUSSION}

Before the observational results can be compared with the values in Table II to yield upper limits on the debris cloud masses, three points should be discussed: (1) the uncertainty in $r_{\text {min }}$ in Eqs. (2), (4), and $(6),(2)$ the uncertainty in the normal $\mathrm{N}$ magnitudes, and (3) the opacity of the debris clouds.

It may be argued that $r_{\text {min }}$ should be about 0.5 to $1 \mu \mathrm{m}$ because radiation pressure would tend to blow such particles out of the solar system. Calculations by Soter et al. (1977) show that for a limited range of 
TABLE III

Ursa Major Stream Stars Observed in 8 to $13-\mu \mathrm{m}$ Band

\begin{tabular}{|c|c|c|c|c|c|c|c|c|}
\hline \multirow[t]{2}{*}{ NGR No. } & \multirow[t]{2}{*}{ BS/HR } & \multirow{2}{*}{$\begin{array}{l}\text { Other } \\
\text { name }\end{array}$} & \multirow[t]{2}{*}{$V$} & \multirow[t]{2}{*}{ Type } & \multirow{2}{*}{$\begin{array}{c}\text { Normal } \\
\mathrm{N}\end{array}$} & \multirow{2}{*}{$\begin{array}{l}\text { Meas. } \\
\mathrm{N}\end{array}$} & \multicolumn{2}{|c|}{ Measured N limits } \\
\hline & & & & & & & l $\sigma$ lower & $1 \sigma$ upper \\
\hline $9^{a}$ & 4931 & $78 \mathrm{UMa}$ & 4.93 & F2V & 4.0 & & 3.7 & $\infty$ \\
\hline 10 & - & HD 115043 & 6.74 & $\mathrm{G} 2 \mathrm{~V}$ & 5.3 & 4.1 & 3.6 & 5.5 \\
\hline 23 & 235 & $\phi^{2} \mathrm{Cet}$ & 5.2 & F8V & 3.9 & & 2.7 & $\infty$ \\
\hline 29 & 330 & 80 Psc & 5.53 & $\mathrm{~F} 2 \mathrm{~V}$ & 4.6 & & 3.3 & $\infty$ \\
\hline $106^{b}$ & 1983 & $\gamma$ Lep A & 3.6 & F6V & 2.5 & & 2.4 & $x$ \\
\hline $106^{b}$ & 1982 & $\gamma$ Lep B & 6.15 & $\mathrm{~K} 2 \mathrm{~V}$ & 4.1 & & 4.6 & $\infty$ \\
\hline 110 & 2047 & $x^{1}$ Ori & 4.41 & GoV & 3.06 & 3.16 & 2.82 & 3.66 \\
\hline 201 & 4623 & $\alpha \mathrm{Crv}$ & 4.03 & F2V & 3.10 & & 1.8 & $\infty$ \\
\hline 236 & 5365 & $18 \mathrm{Boo}$ & 5.41 & F5IV-V & 4.34 & & 4.3 & $\infty$ \\
\hline 243 & 5544 & $\xi$ Boo & 4.54 & G8V & 2.87 & 2.6 & 2.5 & 2.7 \\
\hline 246 & 5634 & 45 Boo & 4.92 & F5V & 3.85 & 3.26 & 2.76 & 4.19 \\
\hline $249^{a}$ & 5727,8 & $\eta$ Cor Bor & 4.9 & G2V & 3.54 & 2.96 & 2.66 & 3.38 \\
\hline 306 & 7172 & $11 \mathrm{Aql}$ & 5.24 & F8IV & 4.0 & 4.3 & 3.9 & 4.7 \\
\hline
\end{tabular}

${ }^{a}$ Double star, both components in field of view.

${ }^{b}$ Part of double star system. Components resolved and measured separately.

particle sizes, about 0.05 to $0.5 \mu \mathrm{m}$, the radiation repulsive force exceeds the force of gravitational attraction for magnetite, graphite, and iron. [See Burns et al. (1979) for a more complete discussion.] For many other materials, including quartz, water ice, obsidian, and andesite, the radiation force does not exceed gravity at any particle size, although it would enable some particles with high kinetic energies to escape that would otherwise have remained in orbit. Thus one would expect a deficit of such particles. The empirical distributions of Le Sergeant and Lamy (1980), in fact, show an inflection in the interplanetary grain number versus size distribution curve in the neighborhood of 1-3 $\mu \mathrm{m}$ which may be related to depletion mechanisms. In a system in which fragmentation is still taking place on a large scale, replenishment of small particles may largely eliminate the deficit. In any case we have partially compensated for the paucity of particles in the vicinity of 1 $\mu \mathrm{m}$ radius by underestimating the number at shorter wavelengths by using $\beta=3.5$. Thus we feel justified in using $r_{\min }$ in the range 0.01 to $0.1 \mu \mathrm{m}$. Particles smaller than
$0.01 \mu \mathrm{m}$ make a negligible contribution to the infrared luminosity because they are absorbing inefficiently $(\xi=\pi r / \lambda)$ throughout the spectral range where the Sun emits most of its energy. For example, it we reduced $r_{\min }$ from 0.01 to 0 in the first example of Table II, the ratio $\Gamma(10.5 \mu \mathrm{m})$ would rise from 3.7 to 4.3 .

The normal values of $\mathrm{N}$ in Table III are based on the V-N values of Johnson (1966) and extrapolations based on effective temperature and the Planck radiation law. Possible uncertainties and the expected spread in these values are not well established. This is not a serious problem for the approach described here because suspected infrared excesses would be examined with 4\% CVF spectroscopy. One Ursa Major Stream system, $\xi$ Boo, was studied this way in the range 8.5 to $13.5 \mu \mathrm{m}$. It was found to have a spectrum consistent with the sum of two blackbodies at the effective temperatures and relative luminosities of its component stars.

In applying Eq. (6) we must be careful to restrict the $f_{j}$ to values small enough so that the inner particles do not shade the outer 
ones enough to significantly lower their temperature. This requires that we calculate the opacity in the ecliptic plane from the center of the toroid system to the outside. The cross-sectional area of all the (spherical) particles in toroid $j$ is

$$
A_{j}=\int_{r_{\min }}^{r_{\max }} \pi r^{2} \frac{d N\left(r, R_{j}\right)}{d r} d r .
$$

Using Eqs. (3), (5), and (7) we have

$$
A_{j}=0.75 f_{j} M_{j} /\left(4 \rho r_{\max }{ }^{1 / 2} r_{\min }^{1 / 2}\right) .
$$

The cross-sectional area of the toroid of major radius $R_{j}$ and minor radius $W_{j}$ as seen from the central star is about $4 \pi R_{j} W_{j}$. The average opacity is thus $A_{j} /\left(4 \pi R_{j} W_{j}\right)$. The ratio of the diameter to the average thickness is $4 / \pi$. Consequently the diametrical opacity is

$$
\tau_{j}=A_{j} /\left(\pi^{2} R_{j} W_{j}\right)
$$

We let

$$
W_{j}=R_{j} \tan 2 \bar{i},
$$

where $i$ is the average inclination to the ecliptic of the asteroids, $9.5^{\circ}$ (Allen, 1973). If we assume $f_{j}=0.1$ for all $j, r_{\min }=0.01$ $\mu \mathrm{m}, r_{\max }=50 \mathrm{~km}$, and other values from Table I and the first example in Table II, then $\Sigma_{j} \tau_{j}=0.25$. It is clear that our present treatment is restricted to $f_{j} \leq 0.1$, particularly for the interior orbits which have the greatest shading effect. Greater opacity would mean lower temperatures for the particles and a less than linear increase in luminosity with $f_{j}$.

The significance of the measured upper limits on $10-\mu \mathrm{m}$ flux may be judged by comparison with the calculated infrared excesses $\Delta M_{\mathrm{N}}$ in Table II. As noted previously, lower limits on $\mathrm{N}$-magnitude (upper limits on $10.5-\mu \mathrm{m}$ flux) of 0.3 below the normal $\mathrm{N}$ values were measured for 6 stars and of 1.7 or less for all 13 stars. From Table II we note that a 0.34 magnitude excess corresponds to a debris cloud of mass $2.2 \times 10^{-6}$ $M_{\odot}(f=0.01)$ for $r_{\min }$ as high as $0.01 \mu \mathrm{m}$ for a G2V star. An excess of 1.7 magnitudes corresponds to roughly 10 times as massive a debris cloud. It should be emphasized that the debris cloud mass-to-star flux ratio depends on our choice of $r_{\max }$ (varying as $r_{\max }{ }^{-1 / 2}$ ) as seen from Eqs. (4), (5), (6), and on the size distribution parameter, $\beta=3$.5. In the case of asteroids, this distribution parameter is constant for sizes up to approximately $r_{\max }=200 \mathrm{~km}$ (based on masses up to $5 \times 10^{19} \mathrm{~kg}$ ) according to Dohnanyi (1969). As noted previously, we used $r_{\max }=$ $50 \mathrm{~km}$ for fragmentation debris on the basis of observed lunar crater sizes. Larger objects are not considered here to be part of the debris cloud. Their mass constitutes the fraction $1-f$ of total planetary mass that has already accreted.

Since the stars listed in Table III are 2.7 $\times 10^{8}$ years old (Giannuzzi, 1979), the apparent lack of debris clouds can be considered to place an upper limit on the time scale of planetary accretion. Theories of gas-free accretion predict time scales on the order of $10^{8}$ years for accumulation of the terrestrial planets (Wetherill, 1980). Safronov's (1969) approach yields $99 \%$ accretion in $1 \times 10^{8}$ years for Earth, and $99 \%$ in $3 \times 10^{8}$ years for Jupiter. In the calculations for Table II, typically $\mathbf{2 0 \%}$ of the luminosity came from the Jupiter toroid, $30 \%$ from the Earth toroid, $40 \%$ from the Venus toroid. Thus the debris in the terrestrial region dominates the luminosity. Consequently, our measurements for the Ursa Major Stream are not capable of ruling out gasfree accretion of planets, but appear to decrease the range of possible time scales $(6 \times$ $10^{7}$ to $6 \times 10^{8}$ years for $98 \%$ accretion) discussed by Safronov (1977) to less than 2.7 $\times 10^{8}$ years. It would be highly desirable to extend this type of measurement to a younger set of stars such as the Pleiades. This will be much more difficult than the present study because of the greater distance. Solar-type stars in the Pleiades are about 5 magnitudes fainter than the stars studied in this work (Crawford and Perry, 1976).

\section{CONCLUSIONS}

The measurements summarized in Table 
III place model-dependent upper limits on the mass of rocky debris that can be present in the vicinity of the stars listed. The actual mass limits depend on the particle size distribution and the spatial distribution of the particles. Using reasonable assumptions based on our knowledge of fragmentation, crater sizes, and removal mechanisms in the solar system, it appears that an N-magnitude excess of 0.34 magnitudes places an upper limit of $2.2 \times 10^{-6} M_{\odot}$ (1\% of the rocky mass of the planets) on the mass of the debris cloud for a solar-type star. Six of the thirteen stars studied fell below this limit. Similarly the upper limit of 1.7 for the $\mathrm{N}$-magnitude excess on all the stars in Table III corresponds to $22 \times 10^{-6} M_{\odot}$ or $10 \%$ of the rocky mass of the planets surrounding a solar-type star. Such limits may be useful in evaluating the extent to which planet formation is taking place in the vicinity of young stars. The existence of observational data lends impetus to the application of more sophisticated models to evaluate the luminosity of the debris clouds produced in different theories of planet formation. It is hoped that the observations will extend to solar-type stars in the Pleiades in order to study an earlier time period when more dust may be present.

\section{AC KNOWLEDGMENTS}

It is a pleasure to thank the staffs of the NASA/ Univ. of Arizona 60-in. Mt. Lemmon Observatory and the Lick Observatory for their help. We thank $H$. Dinerstein for assistance in observations with the Lick Observatory grating spectrometer. We thank P. Cassen, R. Reynolds, D. Hollenbach, and M. Werner for helpful comments.

\section{REFERENCES}

Allen, C. W. (1973). Astrophysical Quantities, 3rd ed., pp. 140, 151. Oxford Univ. Press (Athlone), London/New York.

Brownlee, D. E., F. Horz, F. F. Vedder, D. E. GAUlt, AND J. B. Hartung (1973). Some physical parameters of micrometeoroids. Proc. Lunar Sci. Conf. 4th. 3197-3212.

Burns, J. A., P. L. LAMY, ANd S. Soter (1979). Radiation forces on small particles in the solar system. Icarus 40, 1-48.
Crawford, D. L., and C. L. Perry (1976). Fourcolor $\mathrm{H} \beta$ photometry for open clusters. XI. The Pleiades. Astron. J. 81, 419-426.

DohnanYi, J. S. (1969), Collisional model of asteroids and their debris. J. Geophys. Res. 74, 2531-2554.

Gehrz, R. D., AND N. J. Woolf (1971). Mass loss from M stars. Astrophys. J. 165, 285-294.

GiannuzZı, M. A. (1979). On the eclipsing binaries of the Ursa Major Stream. Astron. Astrophys. 77, 214222.

Gillett, F. C., K. M. Merrill, and W. A. Stein (1971). Observations of infrared radiation from cool stars. Astrophys. J. 164, 83-90.

Greenberg, R. (1979). Growth of large, late-stage planetesimals. Icarus 39, 141-150.

Grossman, A. S., J. B. Pollack, R. T. Reynolds, AND A. L. Summers (1980). The effect of dense cores on the structure and evolution of Jupiter and Saturn. Icarus 42, 358-379.

HACKWELL, J. A. (1972). Long wavelength spectrometry and photometry of M, S, and C-stars. Astron. Astrophys. 211, 239-248.

Hartmann, W. K. (1972). Paleocratering of the Moon: Review of post-Apollo data. Astrophys. Space Sci. 17, 48-64.

HaRTMANN, W. K. (1978). The planet-forming state: Toward a modern theory. In Protostars and Planets (T. Gehrels, Ed.), pp. 58-73. Univ. of Arizona Press, Tucson.

HofFleit, D. (1964). Catalog of Bright Stars, 3rd ed. Yale University Observatory, New Haven, Conn.

Hubbard, W. B., and J. J. McFarlane (1980). Structure and evolution of Uranus and Neptune. $J$. Geophys. Res. 85, 225-234.

Johnson, H. L. (1966). Astronomical measurements in the infrared. Annu. Rev. Astron. Astrophys. 4, 193-206.

Le Sergeant, L. B., and P. H. Lamy (1980). On the size distribution and physical properties of interplanetary dust grains. Icarus $\mathbf{4 3}, 350-372$.

LEUNG, C. M. (1975). Radiation transport in dense interstellar dust clouds. I. Grain temperatures. Astrophys. J. 199, 340-360.

Morrison, D. (1977). Sizes and albedos of the larger asteroids. Icarus 31, 185-220.

Roman, N. G. (1949). The Ursa Major group. Astrophys. J. 110, 205-241.

Safronov, V. S. (1969). Evolution of the Protoplanetary Cloud and Formation of the Earth and Planets. NASA TTF-677. Nauka, Moscow. [Transl. Israel Program for Scientific Translations, 1972]

Safronov, V. S. (1977). Time scale of the formation of the Earth and planets and its role in their geochemical evolution. In NASA SP-370, p. 797.

Soter, S., J. A. Burns, AND P. L. LAMY (1977). Radiation pressure and Poynting-Robertson drag for small spherical particles. In Comets, Asteroids, Meteorites-Interrelations, Evolution, and Origins (A. 
H. Delsemme, Ed.), pp. 121-125. Univ. of Toledo, Toledo, Ohio.

WETHERILL, G. W. (1975). Late heavy bombardment of the Moon and the terrestrial planets. Proc. Lunar Sci. Conf. 5th 1539-1561.

WetheriLl, G. W. (1978). Accumulation of the terres- trial planets. In Protostars and Planets (T. Gehrels, Ed.), pp. 565-598. Univ. of Arizona Press, Tucson. WETHERILL, G. W. (1980). Formation of the terrestrial planets. Annu. Rev. Astron. Astrophys. 18, 77-113. YUAN, C., AND A. M. WAXMan (1977). Kinematic age of moving groups. Astron. Astrophys. 58, 65-78. 